\title{
Evaluación de la resistencia de genotipos de tomate frente a aislados de Phytophthora infestans provenientes de Guatemala
}

\author{
Evaluation of the resistance of tomato genotypes against isolates \\ of Phytophthora infestans from Guatemala \\ Julio E. Berdúo-Sandoval ${ }^{1}$, José A. Ruiz-Chután ${ }^{1,2}$, Amílcar Sánchez-Pérez ${ }^{1}$ * \\ ${ }^{1}$ Facultad de Agronomía, Universidad de San Carlos de Guatemala, Guatemala \\ ${ }^{2}$ Department of Crop Sciences and Agroforestry, Czech University of Life Sciences Prague, Czech Republic
}

*Autor al que se dirige la correspondencia: gramisp@hotmail.com

Recibido: 25 de octubre 2018 / Revisión: 28 de marzo 2019 / Aceptado: 10 de mayo 2019

\section{Resumen}

$\mathrm{E}$ l oomicete Phytophthora infestans (Mont) DeBary es el patógeno responsable de causar la enfermedad deno- $m i n a d a$ comúnmente como tizón tardío. Dicho patógeno afecta cultivos de importancia económica para el país, entre ellos el tomate, cultivo en el cual puede generar pérdidas totales en la producción. Dada la agresividad del patógeno, los programas de mejoramiento desarrollan nuevos genotipos de tomate resistentes a esta enfermedad mediante la incorporación de genes de resistencia, como los genes $P h$. Sin embargo, las nuevas cepas de $P$. infestans, producto de la recombinación genética, pueden sobrepasar la resistencia de los genotipos mejorados. En este estudio se evaluó la resistencia genética de 13 genotipos de tomate mejorados, ante cinco aislados de $P$. infestans obtenidos de un estudio previo. Mediante inoculaciones in vitro y con la variable de respuesta de área bajo la curva del progreso de la enfermedad (AUDPC), se determinó que existió diferencia estadísticamente significativa ( $p<$ 0.05) entre el comportamiento de la resistencia, la agresividad de los aislados del patógeno y la interacción entre ambos factores. Además, se sugiere el uso de algunas líneas para ser incorporadas en programas de mejoramiento genético y así desarrollar nuevos genotipos aptos para el país y, por último, la profundización para establecer las bases genéticas de la resistencia de los dos cultivares evaluados en este estudio.

Palabras claves: tizón tardío, genes $P h$, variabilidad genética, área bajo la curva del progreso de la enfermedad (AUDPC)

\begin{abstract}
$\mathrm{T}$ he oomycete Phytophthora infestans (Mont) DeBary causes late blight disease. This pathogen affects economically important crops for the country, including tomato, a crop in which it can causes total losses in production. Given the aggressiveness of the pathogen, tomato breeding programs develop new genotypes with resistance to $P$. infestans, being a source of resistance $P h$ genes. However, the new strains of $P$. infestans, product of the genetic variability of their populations, can overcome the resistance of the genotypes. In this study, the genetic resistance of 13 tomato materials was evaluated against 5 isolates of $P$. infestans obtained from a previous study. Through in vitro inoculations and with the area under the disease progress curve (AUDPC), it was determined that there was a statistically significant difference $(p<0.05)$ between the behavior of the genetic resistance, the aggressiveness of the isolates of the pathogen and the interaction between both factors. It is also suggested the use of some lines to be incorporated into tomato breeding programs and develop new genotypes suitable for the country and, finally, to establish the genetic basis of the resistance of the two cultivars evaluated in this study.
\end{abstract}

Keywords late blight disease, $P h$ genes, genetic variability, area under the disease progress curve (AUDPC) 


\section{Introducción}

En Guatemala, el cultivo de tomate (Solanum lycopersicum L.) es considerado uno de los cultivos hortícolas de mayor importancia económica, ya que en el 2016 se proyectó una cosecha de 318,181.8 Tm en un área de 8,248.5 ha (Maga, 2016). La producción de tomate trae consigo el reto del manejo de plagas, enfermedades, malezas y condiciones climáticas adversas. De esta lista de factores, las enfermedades representan la mayor complicación, al estimarse más de 200 alrededor del mundo capaces de afectar la producción del cultivo. Dentro de estas, el tizón tardío, causado por el oomicete Phytophothora infestans (Mont.) DeBary, representa el mayor problema a nivel mundial (Fry, 2008). Este patógeno tiene un devastador impacto económico, ya que puede causar pérdidas de hasta el 100\% de la producción en regiones tropicales y subtropicales (Chowdappa et al., 2013).

Debido a esto, el patógeno ha sido motivo de estudios patológicos y genéticos desde la gran hambruna irlandesa en la década de 1840 , la cual dejó tras de sí la muerte y desplazamiento de millones de personas (Martin et al., 2013; Panthee, Gardner, Ibrahem, \& Anderson, 2015; Stroud, Shaw, Hale, \& Steele, 2016). El continuo estudio del patógeno ha demostrado que en años recientes han surgido nuevas cepas de $P$. infestans más agresivas, dificultando así el manejo de la enfermedad (Cooke et al., 2012) por la resistencia desarrollada al control químico (Hu et al., 2012) debido aparecimiento de líneas clonales (Danies, Small, Myers, Childers, \& Fry, 2013; Lees et al., 2012).

Debido a su capacidad de reproducirse de forma sexual (Gallegly \& Galindo, 1958), existen claros indicios de que el proceso de recombinación ha generado un incremento en su variabilidad genética, provocando cambios en su epidemiología resistencia a fungicidas y adaptación a nuevos factores bióticos y abióticos (Cooke et al., 2011) como lo reportado en las poblaciones del sur de India (Chowdappa et al., 2015) y China (Li et al., 2013).

La latente amenaza de nuevos brotes de $P$. infestans, así como su alcance devastador y la vulnerabilidad del sistema de producción en el cultivo de tomate, quedó demostrada en el 2009 durante la pandemia de tizón tardío ocurrida en el noreste de los Estados Unidos. Esto fue el resultado de condiciones ambientales favorables para el patógeno, la amplia dispersión de plántulas de tomate infectadas a través de distribuidores menores, la falta de conocimiento público sobre los sín- tomas y la presencia de las nuevas líneas clonales US22, US-23 y US24 (Fry et al., 2013; Hu et al., 2012). Entre las estrategias para el control de esta enfermedad, el factor genético juega un papel preponderante, por lo que, desde la década de 1940 , se iniciaron programas de mejoramiento para desarrollar materiales de tomate resistentes a $P$. infestans mediante la identificación e introgresión de genes de resistencia (Richards \& Barrat, 1946; Walter \& Conover, 1952).

El gen dominante de resistencia $P h-1$ fue el primero en ser incorporado en un material de tomate domesticado a partir del pariente silvestre Solanum pimpinellifolium L. Dicho gen le confirió resistencia cualitativa ante la raza 0 de $P$. infestans, pero fue rápidamente superado por nuevos genotipos del patógeno. Este gen se encuentra ubicado en el cromosoma 7 (Gallegly, 1968; Pierce, 1971). El gen incompletamente dominante $P h$-2, ubicado en el cromosoma 10 y proveniente de $S$. pimpinellifolium, confiere resistencia parcial hacia algunos genotipos de $P$. infestans por lo que se asemeja a una resistencia de tipo cuantitativa. Dicho gen tiene una acción de retraso en el desarrollo de la enfermedad, pero no logra detenerla por completo (Moreau, Thoquet, Olivier, Laterrot, \& Grimsley, 1998) y generalmente fracasa ante la presencia de cepas más agresivas (Black, Wang, Hanson, \& Chen, 1996; Goodwin, Schneider, \& Fry, 1995). Este gen fue introducido en la década de 1960 (Gallegly, 1968).

El gen incompletamente dominante $P h-3$ ha demostrado conferir una fuerte resistencia y fue identificado en la década de 1990 por investigadores del World Vegetable Center, Centro Asiático de Investigación y Desarrollo de Vegetales en Taiwán (Black et al., 1996). Desde entonces, este gen ha sido el principalmente utilizado, debido a que la resistencia que presenta es efectiva a un amplio rango de cepas de P. infestans, incluyendo aquellas que han superado la resistencia conferida por los genes $P h-1$ y $P h-2$, desarrollándose importantes materiales como las líneas CULBPT-A46 and CULBPT-A48 (Kim \& Mutschler, 2006), NC 1 CELBR and NC 2 CELBR (Gardener \& Panthee, 2010a) y los híbridos Plum Regal (Gardener \& Panthee, 2010b) y Moutaian Merit (Panthee \& Gardner, 2010). $\mathrm{Se}$ han desarrollado otras caracterizaciones del gen $\mathrm{Ph}$ 3 , a partir de diversas accesiones de $S$. pimpinellifolim, en busca de nuevas formas más efectivas en el control de P. infestans (Kim \& Mutschler, 2005).

Sin embargo, se ha determinado que existen nuevas cepas que han superado la resistencia conferida por el gen $P h-3$ (Chunwongse, Chunwongse, Black, 
\& Hanson, 2002) pero al parecer, la combinación de los genes $P h-2$ y $P h-3$ confieren una fuerte resistencia a muchas más cepas. Por esto, prestigiosos programas de mejoramiento alrededor del mundo, como los de la Universidad Estatal del Norte de Carolina, la Universidad Estatal de Pensilvania, la Universidad de Cornell y el Centro Asiático de Investigación y Desarrollo de Vegetales en Taiwán; han logrado la incorporación exitosa de genes de resistencia a $P$. infestans en nuevos materiales de tomate a través de la combinación de marcadores morfólogicos y la selección asistida por marcadores moleculares. Algunos ejemplos de estos son las líneas NC1CELBR y NC2CELBR las cuales incorporan los genes $P h-2$ y $P h-3$ y los híbridos Plum Regal F1 (Ph-3), Mountain Magic F1 ( $P h-2$ y $P h-3)$ y Moutain Merit F1 ( $P h 2$ y $P h 3)$, que han sido liberados a través del programa de mejoramiento de la Universidad Estatal de Carolina del Norte (Gardener \& Panthee, 2010b; Panthee \& Gardner, 2010; Panthee et al., 2015).

Otros cultivares denominados como heirloom, dentro de los cuales destacan los cultivares Matt's Wild Cherry (Solanum lycopersicum var. cerasiforemes (Dunal) Spooner) y Wapsipinicon Peach (Solanum lycopersicum $\mathrm{L}$.), han manifestado una fuerte resistencia a diferentes cepas de P. infestans. No obstante, las bases genéticas de su resistencia son aún desconocidas, tras no haberse identificado los genes $P h-2$ ni $P h-3$, por lo cual es necesario continuar con la caracterización de estos genotipos, ya que la resistencia podría provenir de genes diferentes a los ya identificados (Hoagland et al., 2015; Seidl Johnson, Jordan, \& Gevens, 2014).

Se planteó esta investigación debido a la importancia del cultivo de tomate para la economía guatemalteca, tras haberse demostrado la alta diversidad genética de $P$. infestans en el país (Ruiz-Chután, Berdúo-Sandoval, \& Sánchez-Pérez, 2018) y luego de la identificación de cepas cada vez más agresivas, que han sobrepasado la resistencia de muchos variedades mejoradas (Foolad, Merk, \& Ashrafi, 2008; Foolad, Sullenberger, Ohlson, \& Gugino, 2014; Fry et al., 2015; Yin et al., 2017). El objetivo fue evaluar la resistencia de seis líneas puras de tomate, cinco híbridos y dos cultivares contra la infección de cinco aislados de $P$. infestans. Dichos aislados representan los grupos filogenéticos identificados en Guatemala, determinados en un estudio previo (Ruiz-Chután et al., 2018). Para la evaluación se colectaron muestras de tejido vegetal con los síntomas típicos causados por P. infestans en plantaciones de tomate y papa en Guatemala, a partir de las cuales se aisló el patógeno y posteriormente se realizaron los ensayos de inoculación sobre los genoti- pos de tomate. Se tomaron lecturas de severidad sobre las hojas afectadas y se calculó el área bajo la curva del progreso de la enfermedad, como parámetro para determinar la efectividad de la resistencia de los genotipos evaluados.

\section{Materiales y métodos}

\section{Colecta y aislamiento de $P$. infestans}

Debido a que el patógeno no discrimina en su ataque entre plantas de tomate y papa, se decidió realizar el aislamiento de este a partir de ambos cultivos. Se colectaron 45 muestras de tejido foliar de plantas de tomate y 20 de tejido foliar de plantas de papa durante los meses de mayo a septiembre, periodo en el cual prevalecen las condiciones de alta humedad, temperaturas frescas y periodos nublados en las zonas productoras de tomate y papa en Guatemala, las cuales favorecen el desarrollo del patógeno según Rick y Chetelat (1995). A partir de estas se logró el aislamiento del patógeno en siete muestras de tomate y 15 de papa. Los detalles sobre la procedencia de los sitios de colecta se presentan en la Tabla 1.

Para la evaluación de resistencia se emplearon únicamente los aislados identificados como Patzicía (P10), Sanarate (P16), San Juan Ostuncalco (P17), San Marcos (P19) y Todos Santos Cuchumatán (P22) por representar los grupos filogenéticos identificados en algunas de las principales zonas productoras de tomate y papa de Guatemala en un estudio previo (Ruiz-Chután et al., 2018)

En cada parcela se colectaron 10 hojas afectadas por $P$. infestans procurando identificar los síntomas iniciales de la enfermedad para reducir el riesgo de contaminación provocados por patógenos secundarios. Tras el traslado de las muestras al laboratorio de Biotecnología Vegetal de la Facultad de Agronomía de la Universidad de San Carlos de Guatemala, se realizó un proceso de desinfección a las hojas a través de inmersiones en etanol al $70 \%$, hipoclorito de sodio $1.25 \%$ y agua destilada estéril.

Luego, las hojas fueron sembradas en placas de Petri con medio de cultivo sólido de arveja suplementado con $100 \mathrm{ppm}$ de penicilina y $30 \mathrm{ppm}$ de procloraz e incubadas en oscuridad a $20^{\circ} \mathrm{C}$. Se obtuvieron cultivos puros a partir de zoosporas únicas. Para propiciar el desarrollo del oomicete, se realizaron resiembras a intervalos de 4 semanas empleando para ello el medio de cultivo de arveja sin la adición de ningún antibiótico. 
Tabla 1

Aislados de P. infestans utilizados en este estudio y su procedencia de algunas zonas productoras de tomate y papa de Guatemala

\begin{tabular}{|c|c|c|c|c|c|}
\hline Aislado & Lugar de colecta & Cultivo & Departamento & \multicolumn{2}{|c|}{ Coordernadas } \\
\hline P10 & Patzicía 1 & Papa & Chimaltenango & 14.640814 & -90.940335 \\
\hline P16 & Sanarate & Tomate & El Progreso & 14.81018 & -90.213296 \\
\hline $\mathrm{P} 17$ & San Juan Ostuncalco & Papa & Quetzaltenango & 14.866048 & -91.602249 \\
\hline P19 & San Marcos 1 & Papa & San Marcos & 15.022028 & -91.798506 \\
\hline $\mathrm{P} 22$ & $\begin{array}{l}\text { Todos Santos } \\
\text { Cuchumatán }\end{array}$ & Papa & Huehuetenango & 15.456613 & -91.501255 \\
\hline
\end{tabular}

\section{Selección de genotipos de tomate}

Debido a que en Guatemala no existen híbridos comerciales con resistencia genética al tizón tardío, se seleccionaron genotipos basados en los siguientes criterios, (a) presencia documentada de genes de resistencia y (b) datos publicados que indican la resistencia del genotipo al tizón tardío. Los genotipos seleccionados se comercializan en Estados Unidos. Las semillas fueron compradas en las siguientes compañías distribuidoras de semillas Matt's Wild Cherry, Defiant PHR F1, Mountain Magic, Moutain Merit F1 y Plum Regal en Johnny's Selected Seeds, Waterville, ME; Iron Lady y Wapsipinicon Peach, en High Mowing Organic Seeds, Wolcot, VT, y las semillas NC1CELBR1, NC1CELBR2, NC2CELBRa, NC2CELBRb, NC25Pa, NC25Pb, fueron proporcionados por el Dr. Dillip Panthee, de la Universidad Estatal de Carolina del Norte. Las plántulas del híbrido Retana, que fue utilizado como control susceptible, fueron compradas en la empresa Pilones de Antigua, S.A. El detalle de los genotipos puede consultarse en la Tabla 2.

\section{Preparación del inóculo}

A partir de cada caja de Petri que contenía el oomicete en desarrollo, se agregaron $5 \mathrm{ml}$ de agua destilada estéril y cuidadosamente se realizó un raspado sobre el medio con una espátula de vidrio, colocando la suspensión de esporangios dentro de un vaso de precipitar estéril. La suspensión se ajustó a una concentración de 50,000 esporangios por $\mathrm{ml}$ como sugiere Seidl Johnson y colaboradores (2014) y Akhtar y colaboradores
(2012) y se incubó por dos horas a $10^{\circ} \mathrm{C}$ en condiciones de oscuridad para promover la liberación de zoosporas (Nelson, 2006).

\section{Ensayo para evaluación de resistencia genética a aislados de $P$. infestans}

Los genotipos de tomate se evaluaron utilizando la técnica de cámara húmeda en un diseño experimental completamente al azar bifactorial con tres repeticiones, siendo los factores evaluados los genotipos de tomate (14 niveles) y los aislados de P. infestans (5 niveles) más un control negativo,siguiendo la metodología propuesta por Seidl y colaboradores (2014). Cada repetición consistió de una caja de Petri de 90 $\mathrm{mm}$ de diámetro, conteniendo papel toalla húmeda. En cada caja Petri fue colocada una hoja de cada genotipo, por lo cual el experimento consistió de 252 unidades experimentales.

Las semillas de cada genotipo fueron sembradas en bandejas conteniendo peatmoss como sustrato, las cuales fueron ubicadas en un invernadero libre de $P$. infestans. Tras 5 semanas se procedió a trasplantar 20 plántulas de cada genotipo hacia un invernadero en la Facultad de Agronomía, Usac. Dos semanas después del trasplante se colectaron hojas bien formadas de la parte media de la planta y estas se colocaron en cajas de Petri donde fueron inoculadas haciendo una aplicación de $10 \mu 1$ de suspensión de esporas de P. infestans al centro del haz de cada hoja. Como control susceptible se empleó la variedad Retana, y como control negativo del patógeno se empleó agua destilada. Después de la inoculación, las cajas de Petri fueron colocadas en 
Tabla 2

Descripción genética y morfológica de los materiales de tomate utilizados para pruebas de resistencia genética a $\mathrm{P}$. infestans

\begin{tabular}{clllll}
\hline No. & \multicolumn{1}{c}{ Nombre } & & Bases de resistencia genética & Hábito crecimiento & Color fruto \\
\hline 1 & Defiant PHR F1 & Híbrido & Heterocigoto $P h 2$ y $P h 3$ & Determinado & Rojo \\
2 & Mountain Merit F1 & Híbrido & Heterocigoto $P h 2$ y $P h 3$ & Determinado & Rojo \\
3 & Plum Regal F1 & Híbrido & Heterocigoto $P h 3$ & Determinado & Rojo \\
4 & Iron Lady F1 & Híbrido & Homocigoto $P h 2$ y $P h 3$ & Determinado & Rojo \\
5 & NC1CELBR1 & Linea & Homocigoto $P h 2$ y $P h 3$ & Determinado & Rojo \\
6 & NC1CELBR2 & Linea & Homocigoto $P h 2$ y $P h 3$ & Determinado & Rojo \\
7 & NC2CELBRa & Linea & Homocigoto $P h 2$ y $P h 3$ & Determinado & Rojo \\
8 & NC2CELBRb & Linea & Homocigoto $P h 2$ y $P h 3$ & Determinado & Rojo \\
9 & Matt's Wild Cherry & Cultivar & Desconocido & Indeterminado & Rojo \\
10 & Mountain Magic F1 & Híbrido & Heterocigoto $P h 2$ y $P h 3$ & Indeterminado & Rojo \\
11 & Wapsipinicon Peach & Cultivar & Desconocido & Indeterminado & Amarillo \\
12 & NC25Pa & Linea & Homocigoto $P h 3$ & Determinado & Rojo \\
13 & NC25Pb & Linea & Homocigoto $P h 3$ & Determinado & Rojo \\
14 & Retana & Híbrido & & Determinado & Rojo \\
\hline
\end{tabular}

un incubador a $20^{\circ} \mathrm{C}$ para favorecer el desarrollo de la enfermedad. Las lecturas para evaluar la resistencia de los genotipos de tomate se realizaron a $\operatorname{los} 3,4,5$ y 7 días a partir de la inoculación y mediante el uso de un estereoscopio, se procedió a identificar la presencia de las estructuras del oomicete (micelio y esporangios), así como el área de la lesión provocado por $P$. infestans (Figura 2).

\section{Análisis de datos}

Los datos de área de lesión fueron relacionados con el área foliar total para transformarlos en datos de severidad según el siguiente modelo, severidad = (área de lesión/área foliar total)*100. Con los datos de severidad se calculó el área bajo la curva de progreso de la enfermedad, AUDPC por sus siglas en inglés, ya que, por tratarse de una enfermedad policíclica, este es el parámetro de medición que mejor se ajusta (de la Cruz, Lozoya-Saldaña, Sahagún-Castellanos, \& Peña-Lomelí, 2014). Con los datos obtenidos se realizó un análisis de varianza con un nivel de significancia del $5 \%$ en base al diseño experimental descrito anteriormente, seguido de una comparación de medias mediante la prueba de Tukey con un nivel de significancia del $5 \%$.

\section{Resultados}

Tras realizarse el análisis de varianza se determinó que existió diferencia estadísticamente significativa entre el comportamiento de los aislados de P. infestans, los genotipos de tomate evaluados y la interacción entre el patógeno y los genotipos bajo evaluación, con un $p<.001$ para todos los casos.

Se identificó que los valores AUDPC son variables y están en función al aislado del patógeno utilizado para la inoculación. Para el primer caso, en el cual la inoculación se llevó a cabo con el aislado P10, la comparación de medias mediante la prueba de Tukey generó seis grupos o tipos de comportamiento, siendo los cultivares Wapsipinicon Peach, Matt's Wild Cherry y las líneas NC2CELBRa, NC2CELBRb, NC1CELBR1 y NC25Pa los menos afectados. Las líneas NC1CELBR2, $\mathrm{NC} 25 \mathrm{~Pb}$ fueron clasificados en un segundo grupo. Los híbridos Mountain MeritF1 y Mountain Magic F1conformaron un tercer grupo. El híbrido Iron Lady F1 se colocó en un cuarto grupo. Los híbridos Plum Regal y Defiant PHR F1quedaron en un quinto grupo. Finalmente, el híbrido comercial Retana en el sexto grupo como el más susceptible (Figura 1A). 

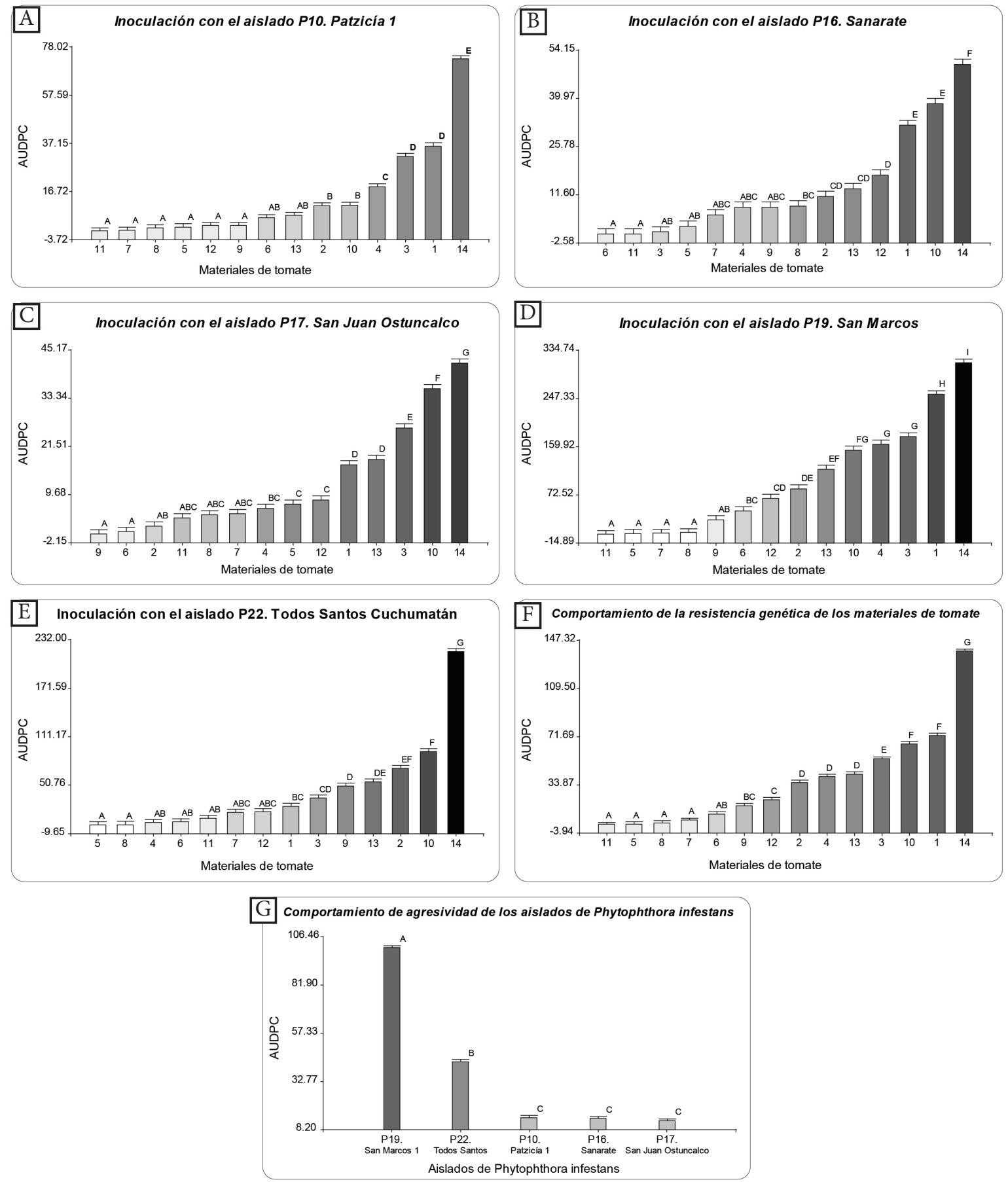

Figura 1. A, B, C, D, E muestra los valores de las pruebas de medias de Tukey del área bajo la curva del progreso de la enfermedad (AUDPC) producto de la inoculación con aislados de Phytophthora infestans de distintas procedencias de Guatemala. F muestra el comportamiento de la resistencia genética de los materiales de tomate contra todos los aislados de P. infestans. G muestra el comportamiento de la agresividad de los aislados de P. infestans contra todos los materiales de tomate. Letras diferentes indican diferencia estadísticamente significativa $p<.05$. La identificación de los materiales de tomate puede observarse en la Tabla 2. 


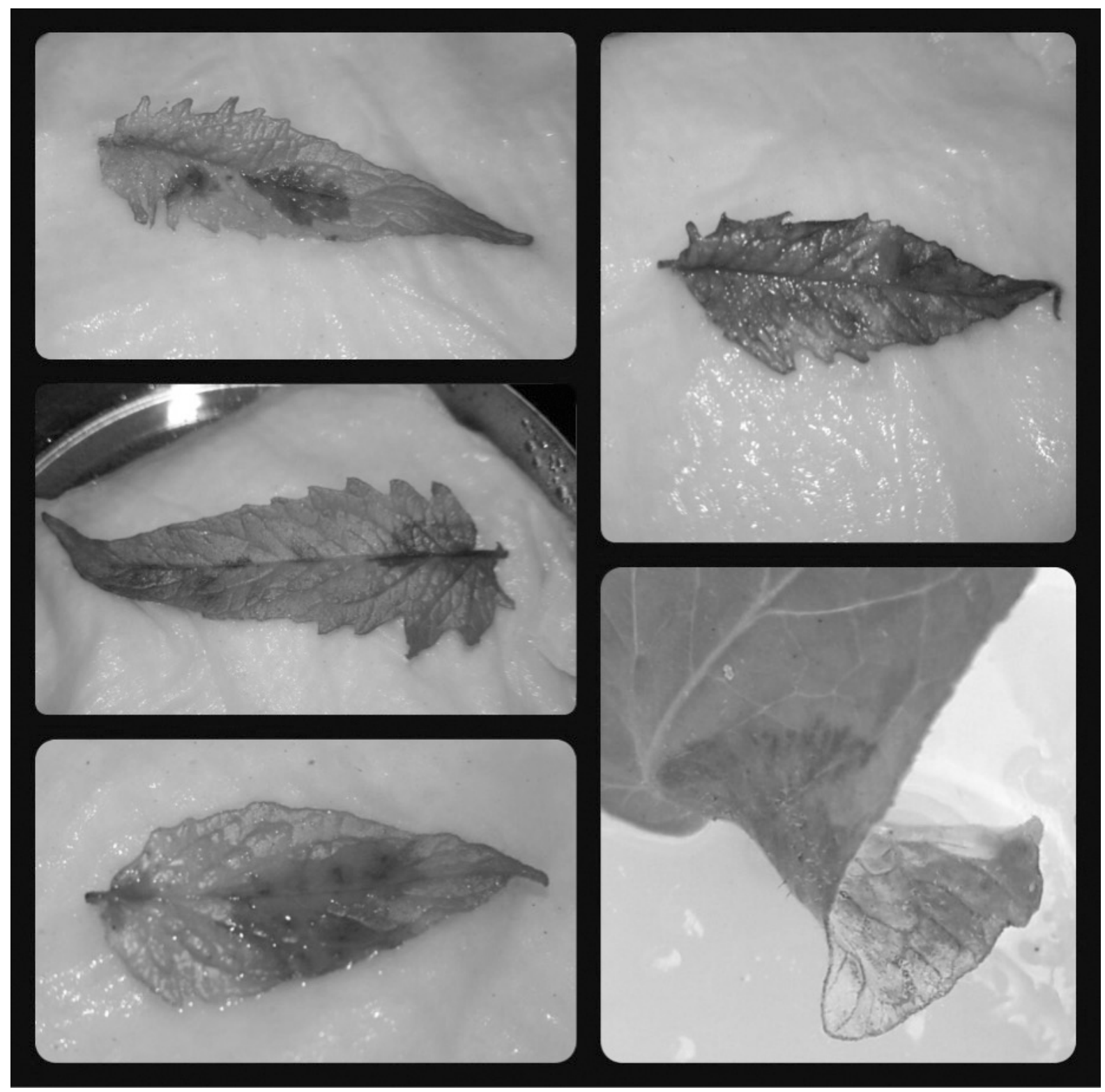

Figura 2. Desarrollo de la enfermedad del tizón tardío sobre hojas de tomate durante los ensayos de inoculación con los aislados de Phytophthora infestans.

En el segundo caso, se utilizó el aislado P16 (Figura 1B), los genotipos fueron clasificados en ocho grupos según la prueba de Tukey. Sin embargo, el orden de agrupación fue distinto al primer caso, siendo los genotipos con los valores más bajos de AUDPC el cultivar Wapsipinicon Peach y la línea NCICELBR2, seguido del segundo grupo con híbrido Plum Regal F1 y la línea NC1CELBR1. El tercer grupo agrupó la linea NC2CELBRa, el híbrido Iron Lady F1 y el cultivar
Matt's Wild Cherry. El cuarto grupo quedó conformado con la línea NC2CELBRb. En el quinto grupo se encontró el híbrido Moutain Merit F1 y la línea NC25Pb. En

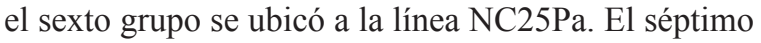
grupo quedó compuesto por los híbridos Defiant PHR F1 y Mountain Magic F1. En el octavo y último grupo se ubicó nuevamente el híbrido Retana.

Los datos obtenidos de la inoculación realizada con el aislado P17, permitió realizar una clasificación 
de nueve grupos tras la prueba de Tukey. Los menores valores de AUDPC corresponden al cultivar Matt's Wild Cherry y la línea NCCELBR2, mientras que los genotipos más afectados fueron los híbridos Mountain Magic F1 y Retana. El comportamiento del resto de genotipos puede observarse en la Figura 1C.

En el caso de las inoculaciones con el aislado P19, se generaron 10 grupos en base a la prueba de media usando la prueba de Tukey, siendo los genotipos con los menores valores de AUDPC el cultivar Wapsipinicon Peach y las líneas NC1CELBR1, NC2CELBRa y NC2CELBRb. Los genotipos mayormente afectados y por lo tanto con los mayores valores de AUDPC fueron los híbridos Defiant PHR F1 y Retana. La distribución del resto de genotipos puede observarse en la Figura 1D.

En el caso del comportamiento de las inoculaciones con el aislado P22, la prueba de Tukey clasificó a los genotipos en diez grupos, siendo los menos afectados las líneas NC1CELBR1, NC1CELBRb, NC1CELBR2, el híbrido Iron Lady F1 y el cultivar Wapsipinicon Peach; mientras que el híbrido Retana fue de nuevo el más afectado. La distribución del resto de genotipos puede observarse en la Figura 1E.

Con la prueba de Tukey también se determinó que los genotipos que mostraron los valores más bajos de AUDPC contra todos los aislados de P. infestans fueron el cultivar Wapsipinicon Peach y las líneas NC1CELBR1, NC2CELBRb y NC2CELBRa. Seguido hubo un segundo grupo donde se encontró la línea NC1CELBR2, luego un tercer grupo compuesto del cultivar Matt's Wild Cherry, un cuarto grupo con la línea NC25Pa, un quinto grupo con los híbridos Moutain Merit F1, Iron Lady F1, y la línea NC25P. El sexto grupo se conformó únicamente por el híbrido Plum Regal F1, un séptimo grupo con los híbridos Mountain Magic F1 y Defiant PHR F1. Por último, se encontró el híbrido Retana, como se observa en la Figura 1F. El análisis de varianza también indicó que existió diferencia estadísticamente significativa en los valores de AUDPC entre los diferentes aislados de $P$. infestans utilizados en las pruebas de inoculación. Los aislados fueron clasificados en 3 grupos según la prueba de comparación de medias de Tukey, en la cual el aislado P19 se ubicó como primer grupo por mostrar los valores más altos de AUDPC y por lo tanto la mayor agresividad. Seguido se encontró el aislado P22 como un segundo grupo de Tukey y finalmente los aislados P17, P16 y P10 se agruparon juntos por poseer los valores más bajos de AUDPC, como puede observarse en la Figura 1G.

\section{Discusión}

Los ensayos de inoculación, para evaluar la resistencia de los genotipos de tomate ante la inoculación de distintos aislados de $P$. infestans, mostraron que, dichos genotipos respondieron de formas diferentes según el grupo filogenético de los aislados con los cuales se realizaron las inoculaciones. De esta forma se reafirman que la diversidad genética de $P$. infestans es un factor crucial en el desarrollo de nuevas poblaciones cada vez más agresivas (Cooke et al., 2012; Danies et al., 2013; Foolad et al., 2008, 2014; Fry et al., 2015; Hu et al., 2012; Lees et al., 2012). Al profundizar en el comportamiento de los diversos genotipos, se puede observar que tras la inoculación realizada con el aislado P10, el cultivar Wapsipinicon Peach fue el que mostró la mayor resistencia frente a $P$. infestans por haber presentado el menor valor de AUDCP.

A pesar de esto, dicho cultivar no presentó el mismo nivel de resistencia cuando fue inoculado con el aislado P17, lo que deja claro que el factor de diversidad genética del patógeno afecta de formas diferentes al mismo genotipo del huesped. Aunque este genotipo fue clasificado en todas las evaluaciones dentro del grupo de Tukey con los genotipos más resistentes, es evidente que su respuesta ante el ataque del patógeno estuvo en función del grupo filogenético del patógeno. Cabe mencionar que la base genética de la resistencia del cultivar Wapsipinicon Peach no está definida, coincidiendo con otros estudios similares en los cuales se determina que la resistencia observada no depende únicamente de los genes $P h-2$ y/o $P h$ - 3 . Esto es debido a que otros híbridos evaluados, que contienen uno o ambos genes, nunca mostraron el mismo nivel de resistencia ante ninguno de los aislados del patógeno (Hoagland et al., 2015; Seidl et al., 2014). No obstante, este cultivar debería ser considerado como un candidato para un programa de mejoramiento para resistencia frente a las poblaciones del oomicete propias del país.

En el caso del híbrido Plum Regal F1, el cual tiene como base de su resistencia al gen $\mathrm{Ph}-3$ (Gardener \& Panthee, 2010b), este fue clasificado dentro de los grupos que mostraron un bajo nivel de resistencia ante los aislados P10, P17 y P19, indicando que, la resistencia genética del gen $P h-3$ puede ser superada por nuevas cepas del patógeno cuando no se acompaña de otro tipo de genes $P h$ (Chunwongse et al., 2002). Sin embargo, este mismo híbrido manifestó un comportamiento interesante al mostrar una resistencia de nivel medio cuando fue inoculado con el aislado P22, siendo este 
un aislado agresivo, ya que ocupó el segundo lugar, en cuanto a la agresividad, en las pruebas de inoculación (Figura 1G).

Por otra parte, este mismo híbrido fue clasificado en el grupo de los genotipos más resistentes cuando fue inoculado con el aislado P16, lo que demuestra, nuevamente, que la resistencia de cada genotipo se ve afectada de forma diferente por el genotipo del patógeno. Al examinar el pedigrí del hibrído Plum Regal F1, se observó que, la línea NC25P, la cual posee el gen de resistencia $P h-3$ en estado homocigoto, fue utilizada como uno de los parentales y, esta misma, tras las pruebas de inoculación en su modalidad $\mathrm{NC} 25 \mathrm{~Pa}$ y $\mathrm{NC} 25 \mathrm{~Pb}$ como líneas hermanas, manifestaron una mayor resistencia en comparación al híbrido Plum Regal F1 ante los aislados P10, P17 y P19. Aún así, estas líneas, $\mathrm{NC} 25 \mathrm{~Pa}$ y $\mathrm{NC} 25 \mathrm{~Pb}$, fueron más susceptibles que el híbrido Plum Regal F1 ante el aislado P16, por lo cual, se asume que el estado de los alelos del gen de resistencia $P h-3$, ya sea en estado de dominancia o recesividad, también influye en la respuesta de la planta ante el ataque del patógeno junto al factor de diversidad genética del patógeno.

El comportamiento de los híbridos Mountain Merit F1 y Moutain Magic F1, los cuales poseen los genes de resistencia $P h-3$ y $P h-2$ provenientes del parental NC1CELBR no fue el mismo. Esto se debió a que el híbrido Moutain Magic F1 fue siempre más susceptible, con excepción de los resultados mostrados tras la inoculación con el aislado P16, en el cual ambos híbridos mostraron el mismo nivel de resistencia. Este resultado contrasta con los datos obtenidos por Hansen, Small, Mutschler, Fry y Smart (2014) quienes clasifican a ambos híbridos dentro del mismo nivel de resitencia. Cabe mencionar que, la inoculación realizada en dicho estudio, se llevó a cabo con la línea clonal US-23 de $P$. infestans, dejando fuera el factor de diversidad genética de las poblaciones del patógeno. Este factor, sí fue tomado en cuenta en esta evaluación, ya que, según Chen y colaboradores (2014), puede llevar a que la resistencia conferida pueda ser superada, en gran medida, por la fluctuación de las poblaciones a causa de la reproducción sexual, como también menciona Vetukuri y colaboradores (2012).

El nivel de resistencia mostrado por los híbridos Mountain Merit F1 y Moutain Magic F1, también es considerado como similar por Ozores-Hampton (2014), quien a su vez menciona que el híbrido Plum Regal F1, a pesar de poseer solamente el gen $P h-3$ como base de su resitencia, muestra niveles más altos de resistencia en comparación a otros genotipos que poseen los genes $P h-2$ y $P h 3$. Esta situación es concordante con este estudio, ya que el híbrido Plum Regal F1 resultó ser más resistente que el híbrido Defiant PHR F1 (Ph-2 y Ph-3) como se observa en la Figura 1F. Otros estudios recientes también han encontrado comportamientos similares en cuanto a la diferencia en los niveles de respuesta de resistencia en función al aislado de $P$. infestans utilizado en las evaluaciones (Ramadan et al., 2017).

En el caso de las líneas NC1CELBR y NC2CELBR mostraron una alta resistencia al ataque del patógeno (Gardener \& Panthee, 2010a), y a su vez, tuvieron un nivel de resistencia más alto que los híbridos generados a partir de ellas, como es el caso de los híbridos Mountain Merit F1 y Moutain Magic F1. Uno de los genotipos que destactó por su alto nivel de resistencia ante todos los aislados de P. infestans fue el cultivar Matt's Wild Cherry, que corresponde a la especie S. lycopersicum var. cerasiforme, del cual no se tiene definida la base de su resistencia genética, ni existen registros que sugieran el mejoramiento de este cultivar. Sin embargo, se puede asumir que, debido a la exposición y desarrollo de este cultivar en las cercanías del valle de Toluca, que es considerado como centro de diversidad secundaria de esta especie (Ferreira, Wetzel, Valois, \& Macedo, 2005), donde también se ubica el centro de origen del patógeno (Goodwin, Spielman, Matuszak, Bergeron, \& Fry, 1992; Goss et al., 2014; Gründwald et al., 2001), puede ser la razón del desarrollo de su alta resistencia debido a la exposición a diversas poblaciones de $P$. infestans (Seidl et al., 2014).

$\mathrm{Al}$ observar el nivel de daño causado por los diferentes aislados empleados para las inoculaciones, es evidente que el más agresivo es el aislado P19 seguido por el aislado P22. Estos aislados fueron clasificados en grupos de Tukey diferentes, a pesar que ambos pertenecen al mismo grupo filogenético, aunque con un valor de similitud de 0.75 por la alta diversidad genética de las poblaciones determinada en un estudio anterior (Ruiz-Chután et al., 2018). El resto de aislados se agruparon juntos en un tercer grupo menos agresivo. Este comportamiento concuerda con los supuestos que el nivel de resistencia de los genes $P h$, es afectado por la composición de las razas de $P$. infestans presentes en una población, así como la herencia poligenética del oomicete (Harbaoui, Harrabi, Vleeshouwers, \& Hamada, 2011; Pule et al., 2013; Tian et al., 2016).

Finalmente, es importante recalcar que en el mejoramiento del tomate para resistencia al tizón tardío, se debe tomar en cuenta la diversidad de las poblaciones 
de $P$. infestans presentes en el área en la cual se establacerá el cultivo. Esto es debido a que la resistencia, conferida por uno o más genes $P h$, puede ser superada por el desarrollo de nuevas cepas cada vez más agresivas, principalmente en zonas donde la diversidad genética del patógeno ya ha sido demostrada. Debido a que los cultivares Wapsipinicon Peach y Matt's Wild Cherry mostraron una alta resistencia a la mayoría de aislados de $P$. infestans evaluados en este estudio, es importe profundizar en la base genética de su resistencia, debido a que no se tiene certeza sobre la misma y ambos genotipos muestran buen potencial para ser incorporados en un programa de mejoramiento tendiente a desarrollar genotipos resistentes a las poblaciones de P. infestans presentes en el país.

\section{Agradecimientos}

Esta investigación fue cofinanciada por Digi-Usac-2017, Proyecto: 4.8.63.4.09.

\section{Referencias}

Akhtar, K. P., Saleem, M. Y., Asghar, M., Ali, S., Sarwar, N., \& Elahi, M. T. (2012). Resistance of Solanum species to Phytophthora infestans evaluated in the detached-leaf and whole-plant assays. Pakistan Journal of Botany, 44(3), 1141-1146.

Black, L., Wang, T., Hanson, P., \& Chen, J. (1996). Late blight resistance in four wild tomato accessions: Effectiveness in diverse locations and inheritance of resistance. Phytopathology, 86, S24.

Chen, A. L., Liu, C. Y., Chen, C. H., Wang, J. F., Liao, Y. C., Chang, C. H., ... Chen, K. Y. (2014). Reassessment of QTLs for late blight resistance in the tomato accession L3708 using a restriction site associated DNA (RAD) linkage map and highly aggressive isolates of Phytophthora infestans. PLoS ONE, 9(5), e96417. doi:10.1371/journal. pone.0096417

Chowdappa, P., Kumar, N., Madhura, S., Kumar, M., Myers, K., Fry, W., ... Cooke, D. (2013). Emergence of 13_A2 blue lineage of Phytophthora infestans was responsible for severe out- breaks of late blight on tomato in South West India. Journal of Phytopathology, 161, 49-58. doi:10.1111/ jph.12031
Chowdappa, P., Kumar, N., Madhura, S., Kumar, M., Myers, K., Fry, W., \& Cooke, D. (2015). Severe outbreaks of late blight on potato and tomato in south India caused by recent changes in the Phytophthora infestans population. Plant Pathology, 64, 191-199. doi:10.1111/ppa.12228

Chunwongse, J., Chunwongse, C., Black, L., \& Hanson, P. (2002). Molecular mapping of the Ph-3 gene for late blight resistance in tomato. Journal of Horticultural Science and Biotechnology, 77(3), 281-286. doi:10.1080/14620316.2002.11511493

Cooke, D., Cano, M., Raffaele, S., Bain, R., Cooke, L., Etherington, G., ... Kamoun, S. (2012). Genome analyses of an aggressive and invasive lineage of the Irish potato famine pathogen. PLOS Pathogens, 8(10), e1002940.

Cooke, D., Schepers, H., Hermansen, A., Bain, R., Dradshaw, N., Ritchie, F., ... Nielsen, B. (2011). Epidemiology and integrated control of potato late blight in Europe. Potato Research, 54, 183222.

Danies, G., Small, I. M., Myers, K., Childers, R., \& Fry, W. E. (2013). Phenotypic characterization of recent clonal lineages of Phytophthora infestans in the United States. Plant Disease, 97(7), 873881. doi:10.1094/PDIS-07-12-0682-RE

de la Cruz, J. B. D., Lozoya-Saldaña, H., Sahagún-Castellanos, J., \& Peña-Lomelí, A. (2014). The Pathosystem Solanum tuberosum L.-Phytophthora infestans (Mont.) de Bary in Chapingo, Mexico. Expected, observed, and simulated. American Journal of Potato Research, 91(3), 312-326. doi:10.1007/s12230-013-9351-y

Ferreira, M. A., Wetzel, M. V., Valois, A. C., \& Macedo, J. (2005). El estado del arte de los recursos fitogenéticos en las américas. Agrociencia, 9(1), 85-90.

Foolad, M. R., Merk, H. L., \& Ashrafi, H. (2008). Genetics, genomics and breeding of late blight and early blight resistance in tomato. Critical Reviews in Plant Sciences, 27(2), 75-107. doi:10.1080/07352680802147353

Foolad, M. R., Sullenberger, M. T., Ohlson, E. W., \& Gugino, B. K. (2014). Response of accessions within tomato wild species, Solanum pimpinellifolium to late blight. Plant Breeding, 133(3), 401-411. doi:10.1111/pbr.12172 
Fry, W. (2008). Phytophthora infestans: the plant (and R gene) destroyer. Molecular Plant Pathology, 9, 385-402.

Fry, W. E., Birch, P., Judelson, H., Grünwald, N., Danies, G., Everts, K., ... Smart, C. (2015). Five reasons to consider Phytophthora infestans a reemerging pathogen. Phytopathology, 105(7), 966-981. doi:10.1094/PHYTO-01-15-0005-FI

Fry, W., McGrath, M., Seaman, A., Zitter, T., McLeod, A., Danies, G., ... Smart, C. (2013). The 2009 late blight pandemic in the eastern United States - causes and results. Plant Disease, 97, 296-306.

Gallegly, M. (1968). Genetics of pathogenicity of Phytophthora infestans. Annual Review of Phytopathology, 6, 375-396.

Gardener, R., \& Panthee, D. R. (2010a). NC 1 CELBR and NC 2 CELBR: Early blight and late blight-resistant fresh market tomato breeding lines. HortScience, 45, 975-976.

Gardener, R., \& Panthee, D. R. (2010b). "Plum Regal" fresh-market plum tomato hybrid and its parents, NC 25P and NC 30P. HortScience, 45, 824-825.

Goodwin, S., Schneider, R., \& Fry, W. E. (1995). Use of Cellulose-acetate electrophoresis for rapid identification of allozyme genotypes of Phytophthora infestans. Plant Disease, 79, 1181-1185.

Goodwin, S., Spielman, L., Matuszak, J., Bergeron, S., \& Fry, W. (1992). Clonal diversity and genetic differentiation of Phytophthora infestans populations in northern and central Mexico. Phytopatology, 82, 955-961.

Goss, E., Tabima, J., Cooke, D., Restrepo, S., Fry, W., Forbes, G., ... Gründwald, N. (2014). The Irish potato famine pathogen Phytophthora infestans originated in Central Mexico rather than the Andes. Proceedings of the National Academy of Sciences, 111, 8791-8796.

Gründwald, N., Flier, W., Sturbaum, A., Garay-Serrano, E., van den Bosch, T., Smart, C., ... Fry, W. (2001). Population structure of Phytophthora infestans in the Toluca Valley region of central Mexico. Phytopatology, 91, 882-890.

Hansen, Z. R., Small, I. M., Mutschler, M., Fry, W. E., \& Smart, C. D. (2014). Differential susceptibility of 39 tomato varieties to Phytophthora infestans clonal lineage US-23. Plant Disease, 98(12), 1666-1670. doi:10.1094/PDIS-03-14-0263-RE
Harbaoui, K., Harrabi, M., Vleeshouwers, V., \& Hamada, W. (2011). Virulence patterns of Phytophthora infestans isolates using $\mathrm{R}$ differential set of Solanum demissum: A useful tool to identify pathogen races in Tunisia. Tunisian Journal of Plant Protection Phytophthora, 6(1), 1-10.

Hoagland, L., Navazio, J., Zystro, J., Kaplan, I., Vargas, J. G., \& Gibson, K. (2015). Key traits and promising germplasm for an organic participatory tomato breeding program in the U.S. midwest. HortScience, 50(9), 1301-1308.

Hu, C. H., Perez, F. G., Donahoo, R., McLeod, A., Myers, K., Ivors, K., ... Ristaino, J.B. (2012). Recent genotypes of Phytophthora infestans in the Eastern United States reveal clonal populations and reappearance of mefenoxam sensitivity. Plant Disease, 96(9), 1323-1330. doi:10.1094/ PDIS-03-11-0156-RE

Jeger, M. J., \& Viljanen-Rollinson, S. L. H. (2001). The use of the area under the disease-progress curve (AUDPC) to assess quantitative disease resistance in crop cultivars. Theoretical and Applied Genetics, 102(1), 32-40. doi:10.1007/s001220051615

Kim, M. J., \& Mutschler, M. A. (2006). CULBPT-A46 and CULBPT-A48 series of late blight resistant processing tomato breeding lines. HortScience, 41(1), 263-265.

Kim, M. J., \& Mutschler, M. A. (2005). Transfer to processing tomato and characterization of late blight resistance derived from Solanum pimpinellifolium L. L3708. Journal of the American Society for Horticultural Sciences 130(6), 877-884. doi: 10.21273/JASHS.130.6.877

Lees, A. K., Stewart, J. A., Lynott, J. S., Carnegie, S. F., Campbell, H., \& Roberts, A. M. I. (2012). The effect of a dominant Phytophthora infestans genotype (13_A2) in Great Britain on host resistance to foliar late blight in commercial potato cultivars. Potato Research, 55(2), 125-134. doi:10.1007/ s11540-012-9214-9

Li, Y., van der Lee, T., Zhu, J., Jin, G., Lan, C., Zhu, S., ... Jacobsen, E. (2013). Population structure of Phytophthora infestans in China - Geographic clusters and presence of the EU genotype Blue_13. Plant Pathology, 62, 932-942.

Martin, M. D., Cappellini, E., Samaniego, J. A., Zepeda, M. L., Campos, P. F., Seguin-Orlando, A., ... 
Gilbert, M. T. P. (2013). Reconstructing genome evolution in historic samples of the Irish potato famine pathogen. Nature Communications, 4, 1-7. doi: $10.1038 /$ ncomms 3172

Ministerio de Agricultura, Ganadería y Alimentación. (2016). El Agro en cifras, 2016. Guatemala: Dirección de Planeamiento del Ministerio de Agricultura, Ganadería y Alimentación.

Moreau, P., Thoquet, P., Olivier, J., Laterrot, H., \& Grimsley, N. (1998). Genetic mapping of $P h-2$ , a single locus controlling partial resistance to Phytophthora infestans in tomato. Molecular Plant-Microbe Interactions, 11(4), 259-269. doi:10.1094/MPMI.1998.11.4.259

Nelson, H. E. (2006). Bioassay to detect small differences in resistance of tomato to late blight according to leaf age, leaf and leaflet position, and plant age. Australasian Plant Pathology, 35(3), 297-301. doi:10.1071/AP06020

Ozores-Hampton, M. (2014). Late blight-resistant tomato varieties evaluation. En M. Ozores-Hampton \& C. Snodgrass (Eds). The Florida Tomato Proceedings conference (pp. 11-13). Florida.

Panthee, D. R., \& Gardner, R. G. (2010). "Mountain Merit": A late blight-resistant large-fruited tomato hybrid. HortScience, 45(10), 1547-1548.

Panthee, D. R., Gardner, R. G., Ibrahem, R., \& Anderson, C. (2015). Molecular markers associated with $\mathrm{Ph}-3$ gene conferring late blight resistance in tomato. American Journal of Plant Sciences, 6, 2144-2150. doi:10.4236/ajps.2015.613216

Pierce, L. (1971). Linkage tests with Ph conditioning resistance to race 0 , Phytophthora infestans. Report of the Tomato Genetics Cooperative, 21, 30.

Pule, B. B., Meitz, J. C., Thompson, A. H., Linde, C. C., Fry, W. E., Langenhoven, S. D., ... Mcleod, A. (2013). Phytophthora infestans populations in central, eastern and southern African countries consist of two major clonal lineages. Plant Pathology, 62(1), 154-165. doi:10.1111/j.13653059.2012.02608.x

Ramadan, A. A., Olfat, M. M., Nour, E. K. S., Kenta, S., Said, M. K., \& Mohamed, T. R. (2017). Resistance to Phytophthora infestans in tomato wild relatives. African Journal of Agricultural Research, 12(26), 2188-2196. doi:10.5897/ AJAR2017.12422
Richards, M., \& Barrat, R. (1946). A partial survey of the genus Lycopersicon for resistance to Phytophthora infestans. Plant Disease, 30, 16-20.

Rick, C., \& Chetelat, R. (1995). Utilization of related wild species for tomato improvement. Acta Horticulturae, 4(12), 21-38.

Ruiz-Chután, J. A., Berdúo-Sandoval, J. E., \& Sánchez-Pérez, A. (2018). Diversidad genética de aislados de Phytophthora infestans colectados en zonas productoras de papa y tomate de Guatemala. Ciencia, Tecnología y Salud, 5(2), 14-24.

Seidl Johnson, A. C., Jordan, S. A., \& Gevens, A. J. (2014). Novel resistance in heirloom tomatoes and effectiveness of resistance in hybrids to Phytophthora infestans US-22, US-23, and US-24 clonal lineages. Plant Disease, 98(6), 761-765. doi:10.1094/PDIS-06-13-0674-RE

Stroud, J., Shaw, D., Hale, M., \& Steele, K. (2016). SSR assessment of Phytophthora infestans populations on tomato and potato in British gardens demonstrates high diversity but no evidence for host specialization. Plant Pathology, 65, 334-341. doi: 10.1111/ppa.12407

Tian, Y. E., Yin, J. L., Sun, J. P., Ma, Y. F., Wang, Q. H., Quan, J. L., \& Shan, W. X. (2016). Population genetic analysis of Phytophthora infestans in northwestern China. Plant Pathology, 65(1), 17-25. doi:10.1111/ppa.12392

Vetukuri, R. R., Åsman, A. K. M., Tellgren-Roth, C., Jahan, S. N., Reimegård, J., Fogelqvist, J., ... Dixelius, C. (2012). Evidence for small RNAs homologous to effector-encoding genes and transposable elements in the oomycete Phytophthora infestans. PLoS ONE, 7(12), e51399. doi:10.1371/ journal.pone.0051399

Walter, J., \& Conover, R. (1952). Hereditary resistance to late blight of tomato. Phytopathology, 42, 197-199.

Yin, J., Gu, B., Huang, G., Tian, Y., Quan, J., Lindqvist-Kreuze, H., \& Shan, W. (2017). Conserved RXLR effector genes of Phytophthora infestans expressed at the early stage of potato infection are suppressive to host defense. Frontiers in Plant Science, 8(December), 1-11. doi:10.3389/ fpls.2017.02155 